\title{
Os atores da regulação assistencial no SUS: quem regula a rede?
}

\author{
The actors of the healthcare regulation in the SUS: who regulates the \\ network?
}

Deise Santana de Jesus Barbosa', Maria do Carmo Lessa Guimarães

DOI: $10.1590 / 0103-1104202112903$

\begin{abstract}
RESUMO Este artigo tem como objetivo identificar os atores sociais envolvidos na ação regulatória para acesso aos serviços assistenciais na rede de pré-natal e parto do Sistema Único de Saúde (SUS), em um município brasileiro. Busca-se analisar as características dos pontos de atenção que integram essa rede temática e suas influências na integração sistêmica entre os atores envolvidos no processo regulatório. Esta análise fundamenta-se na teoria da estruturação, segundo a qual a ação humana molda a estrutura social, que, ao mesmo tempo, orienta a conduta do agente humano, estabelecendo a reprodução/transformação de práticas sociais ao longo do tempo. Os elementos são observáveis nas práticas sociais produzidas na interação entre os atores na ação regulatória para o acesso aos serviços de saúde no SUS. Trata-se de um estudo de caso, cuja pesquisa empírica explorou 32 documentos institucionais, 64 entrevistas semiestruturadas com gestores/profissionais e usuárias da rede e dados secundários, coletados entre setembro de 2018 e janeiro de 2019. Os resultados confirmam a existência de diferentes atores sociais envolvidos na regulação do acesso aos serviços de saúde, caracterizando a regulação assistencial no SUS como uma prática social, revelando a complexidade produzida pela interação entre um conjunto de atores sociais envolvidos nesta ação.
\end{abstract}

PALAVRAS-CHAVE Regulação governamental. Assistência à saúde. Teoria social.

ABSTRACT This article aims to identify the social actors involved in the regulatory action for access to healthcare services in the prenatal and childbirth network of the Unified Health System (SUS), in a Brazilian municipality. It seeks to analyze the characteristics of the points of attention that integrate this thematic network and their influences on the systemic integration between the actors involved in the regulatory process. This analysis is based on the theory of structuring, according to which human action shapes the social structure, which at the same time guides the conduct of the human agent, establishing the reproduction/ transformation of social practices over time. The elements are observable in the social practices produced in the interaction between the actors in the regulatory action for access to health services in SUS. This is a case study, whose empirical research explored 32 institutional documents, 64 semi-structured interviews with managers/professionals and users of the network and secondary data, collected from September 2018 to January 2019. The results confirm the existence of different actors social actors involved in the regulation of access to health services, characterizing the healthcare regulation in the SUS as a social practice, revealing the complexity produced by the interaction between a set of social actors involved in this action.

1 Universidade Federal da Bahia (UFBA), Escola de Administração (EA) Salvador (BA), Brasil. deisesjb@gmail.com
KEYWORDS Government regulation. Delivery of health care. Social theory. 


\section{Introdução}

No cenário de desigualdades sociais e econômicas em que o Sistema Único de Saúde (SUS) está inserido, seus principais desafios se referem ao desequilíbrio entre a demanda e a oferta assistencial da rede e à fragmentação da assistência à saúde prestada ${ }^{1}$, motivo que justificou a adoção da regulação assistencial como função estratégica na organização da rede assistencial de saúde.

A polissemia conceitual do termo regulação reflete-se no processo de implementação do SUS, no qual a regulação foi incorporada como estratégia para a consecução dos princípios de integralidade e equidade que orientam a organização do SUS. Ao privilegiar tanto a execução de atividades normativas de regulamentação do mercado quanto a mediação política e gerencial do acesso aos serviços de saúde, a concepção de regulação incorpora as dimensões teóricas fundamentadas pela teoria econômica e pelas ciências sociais ${ }^{\mathbf{2}, 3}$.

A regulação no SUS pode ser entendida como uma prática social, na medida em que é definida como um "conjunto de ações mediatas, de sujeitos sociais sobre outros sujeitos sociais, que facilitam ou limitam os rumos da produção de bens e serviços em determinado setor da economia"4(34), abrangendo o ato de regulamentar e o conjunto de ações implementadas para assegurar o cumprimento dessas regulamentações ${ }^{4}$. No entanto, contrariamente a essa concepção expressa da regulação como uma prática social, o processo regulatório da saúde no SUS vem se desenvolvendo sob o predomínio da normatividade discricionária.

Essa contradição tem sido objeto de discussão na literatura sobre a regulação no SUS. Para Gawryszewski et al.5, o cenário onde se estabelecem os processos de regulação assistencial no SUS é caracterizado por conflitos e tensões. A ação regulatória é uma função de gestão, mas sua atuação se desenvolve na rede assistencial, interligando dimensões do sistema de saúde permeadas por interesses conflitantes. Para Lobato e Giovanella ${ }^{6}$, embora o Estado seja o principal agente da atividade regulatória no SUS, outros atores, como o mercado, os prestadores de serviços e os profissionais de saúde, também possuem seus próprios mecanismos de regulação, e, muitas vezes, com propósitos divergentes dos estabelecidos pelos gestores públicos. Já Cecílio et al. ${ }^{7}$ complementam essa afirmativa, incluindo a atuação dos usuários na produção de outras lógicas regulatórias, para além da regulação governamental, como estratégia de ampliação do acesso aos serviços dos quais necessitam. Segundo Cecílio ${ }^{10}$, essa característica faz com que a regulação assistencial seja um processo em permanente construção, e, por isso, permanentemente instituinte.

Essas contradições observadas no processo regulatório do acesso à saúde, no âmbito do SUS, podem ser analisadas à luz dos postulados da teoria da estruturação, propostos por Anthony Giddens, ao explicar sobre a dualidade da estrutura observada nos sistemas sociais. De acordo com Giddens, a ação humana molda a estrutura social, que, ao mesmo tempo, orienta a conduta desse agente que executa a ação. Para o autor,

ser um agente é ser capaz de exibir (cronicamente, no fluxo da vida cotidiana) uma gama de poderes causais, incluindo o de influenciar os manifestados por outros ${ }^{8(17)}$.

Um ator social é, portanto, um agente da ação, com capacidade de exercer algum tipo de poder para 'fazer uma diferença' com relação a uma dada situação, por meio dos recursos de que dispõe para realizar a ação. Pela sua condição humana, o ator é capaz de refletir sobre o que faz enquanto o faz, de modo contínuo, no contexto da atividade social que realiza, o que Giddens ${ }^{8}$ chama de cognoscitividade, sejam os resultados alcançados esperados ou não.

A agência é a ação humana orientada, e representa a capacidade que $\mathrm{o}$ ator social possui para fazer coisas, indo além das suas intenções. Giddens $^{\mathbf{9 ( 6 5 )}}$ descreve a ação humana como um "fluxo contínuo de condutas" que são 
influenciadas por circunstâncias estruturais produzidas pelo contexto histórico. $\mathrm{O}$ autor refere-se ao contexto em que ação acontece com relação ao espaço e ao tempo que a envolvem, enfatizando que toda forma de ação social sofre a influência de dimensões sociais, culturais, econômicas, que não podem ser negligenciadas numa análise explicativa.

A teoria da estruturação, através dessas concepções, ressalta a complexidade do modus operandi do sistema de saúde brasileiro, destacando a existência dos elementos estruturais e contextuais que evidenciam a sua dinamicidade enquanto prática social. Por isso, autores como Cecílio ${ }^{10}$ defendem que o conceito de sistema de saúde precisa ser relativizado e que a ação regulatória deve ser entendida como resultado da interação entre múltiplos atores (individuais, coletivos, institucionais ou não), que vão produzindo diversificados regimes de regulação do acesso e do consumo dos serviços de saúde ao longo do tempo e sob determinado contexto.

A ação regulatória se caracteriza por procedimentos, métodos ou técnicas hábeis executadas conscientemente por atores sociais utilizando-se das regras vigentes para disponibilizar os recursos assistenciais disponíveis no sistema de saúde. As regras se apresentam nos aspectos normativo e semântico ${ }^{8}$. O aspecto normativo se refere aos modos pelos quais as práticas podem ser executadas e se expressam, no âmbito do SUS, por meio das portarias, dos decretos e contratos estabelecidos numa rede assistencial temática. $\mathrm{O}$ aspecto semântico das regras refere-se ao significado qualitativo e processual das práticas sociais, sendo construídas coletivamente pelos atores em interação reconhecida como saber técnico.

Na perspectiva da teoria da estruturação, as regras não são estáticas, e, por si só, não determinam a ação dos agentes sociais. Elas são interpretadas e alteradas durante a interação entre os atores envolvidos, que agem com base em subjetivações produzidas sob determinado contexto, de acordo com seus conhecimentos e objetivos na rede, e isso contribui para a mudança na sua aplicação"11. As regras geralmente são envolvidas por um processo de contestação, de modo que, embora sejam adotadas para trazer ordem e minimizar as incertezas, a institucionalização de regras revela-se um processo repleto de contradições e ambiguidades ${ }^{\mathbf{1 2}}$. Essa fundamentação ajuda a compreender os estudos de Cecílio ${ }^{10}$, nos quais o autor observa a existência de diferentes 'regimes de regulação' como agenciamentos que operam no cotidiano do sistema de saúde, para além da regulação governamental, e que vão desenhando outros fluxos, novos pontos de conexão e articulação entre os serviços de saúde, dando ênfase à existência de relações sociais sincrônicas passíveis de serem descritas e estudadas.

Este artigo tem como objetivo identificar os atores sociais envolvidos na ação regulatória para acesso aos serviços assistenciais na rede de pré-natal e parto do SUS, em um município brasileiro. Busca-se analisar as características dos pontos de atenção que integram essa rede temática e suas influências na integração sistêmica entre os atores envolvidos no processo regulatório.

\section{Metodologia}

Trata-se de um estudo de caso, ilustrado a partir da ação regulatória produzida na rede assistencial de pré-natal e parto, no âmbito do SUS, em um município brasileiro, orientado pelos pressupostos da pesquisa qualitativa. Utilizou-se como critério para a seleção do município a existência de uma rede complexa e estruturada de serviços de saúde em todos os níveis de complexidade assistencial, oferecendo serviços de atenção básica, serviços de urgência e emergência e maternidades.

A regulação assistencial no SUS, de acordo com sua normativa, desenvolve-se no Complexo Regulador (CR) e em suas unidades operacionais. Por meio da regulação médica, garante-se o acesso baseado em protocolos, classificação de risco e critérios de priorização 
de acesso à saúde. $\mathrm{O}$ ato de regular é uma atribuição legalmente designada ao médico regulador, autoridade sanitária que representa o Estado nesse processo ${ }^{13}$. Este estudo parte do pressuposto de que os mecanismos normativos que vêm sendo adotados pelo SUS não condizem com um processo regulatório que envolve a interação constante de diversos atores, configurando-se uma ação não completamente controlável, podendo produzir possibilidades e constrangimentos à integração sistêmica da Rede de Atenção à Saúde (RAS).

Os estabelecimentos que compõem o escopo da pesquisa para coleta dos dados foram selecionados seguindo a distribuição territorial dos Distritos Sanitários do município estudado, incluindo seu CR, composto pelas centrais (i) de Regulação de Consultas e Exames, (ii) de Regulação de Urgência/Emergência e (iii) de Regulação de Leitos. Entre as unidades assistenciais de saúde, foram incluídos serviços da atenção básica, serviços ambulatoriais que ofertam consultas e exames, serviços de urgência/emergência e as maternidades, contemplando 44 pontos de atenção da rede assistencial de pré-natal e parto.

Os atores sociais envolvidos na ação regulatória, e que conformam a rede assistencial, são os profissionais de saúde - os que solicitam os recursos e os que realizam os atendimentos; os representantes das organizações que proveem os serviços de saúde - públicos e privados; e os usuários ou seus representantes - que demandam os serviços de saúde.

Foi utilizado um conjunto de instrumentos e técnicas de coleta de dados, envolvendo pesquisa documental, análise exploratória de dados secundários e entrevista semiestruturada com gestores/profissionais de saúde da rede assistencial e com usuárias dos serviços de pré-natal e parto do SUS.

As entrevistas foram orientadas por três roteiros semiestruturados, com especificidades para cada categoria de informantes, a saber: reguladores, gestores/profissionais de saúde e usuárias. Em cada ponto de atenção, foi entrevistado o gestor responsável pela unidade ou um profissional envolvido com a assistência de pré-natal, designado pelo gestor, num total de 44 profissionais. Foram entrevistadas gestantes e puérperas encontradas nas unidades no momento das visitas e que se dispuseram a participar da pesquisa, totalizando 20 mulheres usuárias dos serviços de saúde. Com o objetivo de proteger a identidade dos entrevistados, a identificação das entrevistas dos gestores e trabalhadores de saúde foi realizada pela sigla da unidade assistencial onde se realizou a coleta de dados, seguida de uma numeração sequencial. A identificação das usuárias foi realizada utilizando-se a letra $\mathrm{G}$, seguida de uma numeração sequencial. Todas as 64 entrevistas realizadas foram gravadas, com autorização dos entrevistados, transcritas e analisadas juntamente com os documentos e dados secundários levantados na pesquisa.

A pesquisa documental explorou documentos normativos e institucionais que subsidiaram a Política de Regulação em Saúde e da Rede Cegonha, de âmbito municipal e estadual, envolvendo normas do SUS, registros do Fórum da Rede Cegonha e documentos técnicos produzidos sobre o tema, sendo identificados 32 documentos relevantes para o estudo.

Como dados secundários, foi utilizada a base de dados do Cadastro Nacional de Estabelecimentos de Saúde (CNES) para consulta da ficha detalhada dos estabelecimentos de saúde que integraram a pesquisa, observando informações referentes ao tipo de estabelecimento, nível de gestão e natureza jurídica.

A análise dos dados empíricos buscou estabelecer relações dialéticas, por meio da triangulação, entre o material empírico de diferentes fontes de informação e o referencial teórico, considerando o contexto que envolve o fenômeno pesquisado em sua realidade histórica e social ${ }^{14}$.

A pesquisa foi realizada observando os princípios éticos estabelecidos na Resolução $n^{0}$ 466/12, do Conselho Nacional de Saúde, considerando que envolve pesquisa com seres 
humanos através da análise de práticas sociais. O projeto foi submetido ao Conselho de Ética em Pesquisa (CEP) da Escola de Enfermagem da Universidade Federal da Bahia (UFBA), tendo aprovação expedida por meio do parecer de número 2.556.780, em 22 de março de 2018.

\section{Resultados e discussão}

Giddens ${ }^{9}$ defende que um ator social é um agente intencional, dotado de conhecimentos e habilidades para a ação a que se propõe, e a ação humana ocorre em um fluxo contínuo da sua conduta, que $o$ agente monitora reflexivamente, de forma constante. Portanto, ser um agente é ser capaz de fazer a diferença com relação a uma situação, de modo que a ação desse agente envolve poder, enquanto capacidade de transformar. Contudo, considerando o princípio da dualidade da estrutura, os agentes e a estrutura não constituem corpos independentes na ação. Esses elementos se articulam na produção e reprodução das práticas sociais, ${ }^{\mathbf{8} 9}$.

Compreender sobre quem são os agentes da ação regulatória e suas características permite a compreensão das relações de interdependência que eles produzem em interação, que se configuram nas formas de integração predominantes na implementação cotidiana da rede assistencial, e influenciam a garantia do acesso aos serviços de saúde no SUS.

\section{Os atores da regulação assistencial}

Para identificar os atores e compreender sua atuação no processo regulatório no SUS, a rede assistencial de pré-natal e parto foi estudada sob duas perspectivas distintas de observação. A primeira foi produzida pelos gestores/ profissionais das unidades de saúde dos diferentes níveis de complexidade assistencial entrevistados - nessa perspectiva, as usuárias são apresentadas de forma generalizada. Já a segunda perspectiva privilegia as informações fornecidas pelas usuárias encontradas nas unidades visitadas, cuja interação com outros atores busca viabilizar o acesso aos serviços de que necessitam no cotidiano da rede assistencial. Uma síntese dos atores envolvidos na ação regulatória, descritos por esses dois grupos de atores, encontra-se apresentada no quadro 1.

Quadro 1. Atores da ação regulatória segundo perspectivas de observação da rede assistencial

\begin{tabular}{|c|c|}
\hline Gestores/profissionais da rede assistencial & Usuárias da rede assistencial \\
\hline $\begin{array}{l}\text { Um conjunto diversificado de profissionais são reconhe- } \\
\text { cidos como atores da rede, em especial, os profissionais } \\
\text { da atenção básica, das unidades hospitalares e os regu- } \\
\text { ladores. }\end{array}$ & $\begin{array}{l}\text { Um conjunto diversificado de profissionais são reconheci- } \\
\text { dos como atores da rede, em especial, os enfermeiros da } \\
\text { atenção básica e as unidades hospitalares. }\end{array}$ \\
\hline $\begin{array}{l}\text { Os profissionais médicos se destacam no tocante à per- } \\
\text { cepção sobre a capacidade de garantir o acesso à vaga, } \\
\text { considerando a sua prerrogativa técnica de realizar ou } \\
\text { coordenar o recurso assistencial na saúde. } \\
\text { As usuárias não são reconhecidas explicitamente como } \\
\text { atores que viabilizam o acesso por parte dos profissionais } \\
\text { e gestores. }\end{array}$ & $\begin{array}{l}\text { As usuárias se revelam como atores ativos no acesso aos } \\
\text { serviços de que necessitam. }\end{array}$ \\
\hline
\end{tabular}

Fonte: Elaboração própria.

Nas duas perspectivas de observação, verifica-se que a regulação assistencial sofre constrangimentos na medida em que normas e regras não são suficientes para que o acesso aos serviços de saúde seja operado com base nas recomendações do CR. 
Os relatos dos profissionais entrevistados indicam a participação ativa de dois atores sociais, cuja atuação mostrou-se relevante para a definição de estratégias coletivas, que não haviam sido identificados no desenho preliminar da pesquisa. Um deles refere-se aos Distritos Sanitários existentes no município, pensados como mediadores no fluxo das ações da Rede Cegonha. Os distritos foram considerados como agentes reguladores por profissionais entrevistados, a exemplo do que foi narrado pela enfermeira da unidade mista UM1:

A regulação pra mim é o distrito. Hoje em dia o meu vínculo de regulação é o distrito. Porque, se é alto risco, eu tenho que pedir pra eles marcarem. Se é uma paciente que tem algum problema, tipo ela é usuária de drogas, então, além da UBS, ela precisa de um Caps, o distrito é quem marca. Ela tem HTLV, o distrito marca também. É tudo o distrito que faz, através da rede cegonha. O fluxo nosso sempre foi esse, e funciona bem.

$\mathrm{O}$ outro ator que se mostrou atuante no acompanhamento das ações da Rede Cegonha, em especial, no estímulo às ações de prestação de contas por meio do monitoramento e da avaliação das ações e dos serviços desenvolvidos na assistência à gestante no município estudado, é o Ministério Público Estadual, exercendo sua função de controle externo da administração pública e configurando uma ação baseada na legitimação, segundo a teoria da estruturação. Essa atuação foi descrita pela supervisora de enfermagem da maternidade M5:

A reunião do observatório de maternidades, que é com o Ministério Público também é uma coisa importante, porque ela traz uma certa cobrança de posicionamento dos outros órgãos envolvidos. Da própria Sesab, do município, dos serviços de uma maneira geral. Então, isso fortalece.

Apesar de as usuárias não assumirem um protagonismo claro na regulação do acesso aos serviços, segundo a perspectiva dos gestores/profissionais de saúde, na sua fala, esses atores relatam explicitamente a designação de atribuições às gestantes, na busca dos recursos necessários ao cuidado em saúde. Como exemplificado nos relatos abaixo:

aí a gente só orienta ela procurar alguma maternidade com a sacola do menino pra sair com o menino no colo. 'Hoje é o dia, você não pode passar de hoje. Vá, você entra na maternidade e só vai sair com seu filho no colo'. (Gerente da unidade básica USF7).

aí elas ou fazem particular, ou de repente conseguem através de mutirão, que tem uma escola de imagem que faz o mutirão. (Enfermeira da unidade básica UBS4).

Essa situação foi observada, predominantemente, nas Unidades Básicas de Saúde, que, em tese, deveriam se responsabilizar pela continuidade do cuidado às usuárias na rede assistencial. Entretanto, o que se observa é uma desresponsabilização, por parte dos profissionais assistentes, justificada por eles pela limitação dos recursos assistenciais disponíveis no território do município, conforme narrativa da gerente da unidade básica USF4:

ela que marca, mas não é de responsabilidade nossa. A gente encaminha, dá aquela ficha de referência e lá acolhe, ou outra maternidade que foi encaminhada.

Nessa direção da transferência de responsabilidades para outros pontos da rede, registram-se, inclusive, relatos de orientação para que a gestante denunciasse a falta de acesso à ouvidoria do SUS do município, como afirmado por um profissional da USF5 quando questionado sobre as estratégias da unidade para diminuir as dificuldades de encaminhamento: "[...] orientar as pacientes a ligar pra ouvidoria. A gente 'olhe, ligue pra ouvidoria, viu? Dizendo que não conseguiram vagas', essas coisas".

Na perspectiva das usuárias da rede assistencial, os relatos das entrevistadas revelam 
um protagonismo ativo para viabilizar os serviços que não estão tão acessíveis na rede assistencial de pré-natal e parto do município estudado. As trajetórias contadas pelas usuárias mostram sua peregrinação entre os pontos de atenção e a desintegração da rede assistencial, que não tem conseguido capturar as gestantes e viabilizar seu acesso de forma integrada. Os relatos feitos por essas mulheres autorizam afirmar que há uma falência da ação regulatória, no âmbito da RAS, considerando sua prerrogativa de integrar os pontos de atenção à saúde.

Eu fui lá na M9.1, e me encaminharam pra aqui [M9.2]. Aí eu fui na UM9, e eles também me encaminharam pra aqui. (Gestante G9).

Eu fiz tudo pago. Só uma [ultrassonografia obstétrica] que eu fiz agora na M9.2, porque eu tava sentindo dor, aí eu fiz lá. (Gestante G6.1).

Eu dormi lá pra marcar [ultrassonografia obstétrica]. (Gestante G12.2).

Só foi um pouco difícil porque eu tive que madrugar. Só dão 15 fichas por dia [exames laboratoriais]. (Gestante G6.1).

Não foi encaminhamento. Ela me deu a requisição [a médica da UBS], eu fiquei sabendo que lá faz, e aí eu fui pra lá. [...] A ultrassom eu fiz paga porque eu não sabia que na M2 fazia de graça. Aí a próxima eu vou fazer na M2. (Gestante G5.4).

Essas diferentes situações vivenciadas pelas usuárias do SUS confirmam a existência de uma variedade de nuances possíveis entre a aceitação de uma regra normativa e os modos de adaptação de um ator frente às obrigações impostas e suas consequentes sanções na vida social.

A teoria da estruturação auxilia na explicação dessa situação a partir do "caráter negociado das sanções”, atribuído por Giddens ${ }^{\mathbf{9}(95)}$, o qual revela uma característica inerente das interações sociais que valoriza os elementos de significação com relação à ordem normativa, por meio das quais os agentes, dada a sua capacidade de fazer diferente, no contexto da sua ação, podem transgredir, ou não, as prescrições normativas.

O protagonismo das usuárias no sistema de saúde, para garantir o seu acesso aos serviços dos quais necessita, é reconhecido por alguns autores ${ }^{\mathbf{1 5}, 16}$. Constata-se que a condição para um acesso regulado é a interação, via comunicação com as usuárias, assim como a importância de se observar as contradições entre as reivindicações das usuárias e o saber técnico dos profissionais na rede.

As interações estabelecidas entre os atores influenciam as possibilidades de ação na dinâmica dos sistemas sociais, de modo que as multiplicidades de agentes envolvidos na ação regulatória complexifica o contexto em que se desenvolve o acesso à saúde. Depreende-se que essa diversidade de atores, envolvendo gestores, prestadores, trabalhadores e usuários, tanto pode representar possibilidades para a pactuação de ações estratégicas coletivas de articulação como pode se configurar em constrangimento, quando são usadas as relações pessoais para benefício próprio na priorização do acesso, sem observância dos critérios de necessidade e risco.

Desse modo, ainda que a regulação assistencial seja implementada por meio de normativas (leis, portarias, protocolos e fluxos), no cotidiano da rede assistencial, tais esquemas vão sendo relidos e reformulados, considerando principalmente a restrição de recursos assistenciais na rede. Tais configurações também podem resultar tanto em barreiras de acesso, constrangendo a atuação da rede assistencial, como em possibilidades produzidas por modos de organização do acesso mais integrados e flexíveis, adequados às características específicas locais e às necessidades próprias da população naquele território. 


\section{Perfil dos pontos de atenção da rede assistencial de pré-natal e parto: natureza jurídica e tipo de gestão das unidades de saúde}

A coleta de dados desta pesquisa mostrou a diversidade de características jurídico-administrativas entre os prestadores de serviços de saúde de pré-natal e parto no município estudado. Os atores sociais envolvidos na ação regulatória, portanto, representam ou são representados por organizações que possuem características jurídicas e administrativas diferentes. Santos e Merhy ${ }^{17(31)}$ explicam que o "ator social busca regular os serviços de saúde segundo os interesses da sua representação, ou seja, procura direcionar a produção da saúde para os seus macro-objetivos", considerando os contextos histórico e político em que a rede se desenvolve. Desse modo, conhecer as características referentes à natureza jurídica e ao tipo de gestão dos prestadores de serviço integrantes da rede assistencial contribui para a compreensão da forma como estes interagem entre si na ação regulatória para acesso à saúde no SUS.

A natureza jurídica dos prestadores de serviços da rede indica a constituição jurídico-institucional dos estabelecimentos de saúde. O tipo de gestão dos prestadores da rede indica qual ente federado é o gestor do SUS responsável pela programação, pelo processamento e pelo pagamento de determinada unidade de saúde ${ }^{\mathbf{1 8}}$. Entre os estabelecimentos de saúde pesquisados neste artigo, no que concerne à sua natureza jurídica, não existe nenhuma unidade da classe de empresa privada, mas encontram-se representantes de todas as demais classificações. Contudo, a rede estudada possui a participação do setor privado, através das empresas que terceirizam serviços em algumas unidades de saúde do estado e do município. Com relação ao tipo de gestão, a pesquisa contou com representantes em todas as categorias descritas.

A rede assistencial estudada apresenta o seguinte perfil de unidades de saúde: (i) as unidades básicas de saúde são municipais e estão sob gestão pública direta da SMS; (ii) as unidades ambulatoriais e de urgência e emergência são municipais e, em sua maioria, possuem todos ou parte dos serviços terceirizados; e (iii) as maternidades são de gestão Estadual, possuindo unidades públicas de gestão própria do Estado, unidade terceirizada e unidades sem fins lucrativos.

O exemplo mais peculiar da diversidade de representação está na conformação do próprio CR do município, formado por três centrais de regulação. No município estudado, essa composição integra a Central Estadual de Regulação de Leitos - que pertence à gestão estadual -, a Central de Regulação de Urgência/Emergência e a Central Municipal de Regulação Ambulatorial - que funcionam sob gestão municipal.

A divisão de comando no CR contribui para a fragmentação das ações de regulação no território, contribuindo para uma atuação das centrais de forma atomizada e não articulada. Gawryszewski et al. 5 explicam que, numa federação como o Brasil, onde os entes federativos possuem autonomia administrativa, a descentralização de políticas prescinde da ação integrada entre os gestores do sistema nos três níveis de governo. Contudo, essa prática não vem sendo observada na operacionalização do CR em Salvador, contrariando, inclusive, o princípio normativo do comando único estabelecido no Pacto de Gestão do SUS ${ }^{19}$.

Essa caracterização ajuda a delinear o contexto de complexidade da rede assistencial e, por conseguinte, da ação regulatória que se propõe a integrar esse conjunto de atores, envolvendo a existência de atores que apresentam propriedades jurídicas distintas - pública e privada -, e condução por entes federados diferentes - federal, estadual e municipal. Em determinadas situações, existe diversidade de representação dentro de um mesmo estabelecimento de saúde. A exemplo de uma unidade pública de propriedade do governo federal, cuja gestão é estadual. Ou de uma unidade de 
gestão municipal, em que parte dos serviços é terceirizada ao setor privado e parte é executada diretamente pelo município.

A importância destes achados justifica-se na afirmação de Fleury e Ouverney ${ }^{20}$, segundo os quais uma rede de política pública pressupõe a interação de atores múltiplos que interagem constantemente. Contudo, esses atores sociais agem em função dos seus interesses, expectativas e motivações com relação aos demais atores, com base em elementos do contexto que os envolve.

\section{A garantia do acesso aos serviços de pré-natal e parto: quem se responsabiliza?}

Para além dos documentos normativos estabelecidos na política de saúde, o acesso aos serviços assistenciais, analisado a partir dos dados empíricos deste estudo, vem sendo garantido predominantemente pelos atores envolvidos diretamente na prestação do serviço de saúde ofertado. Os próprios gestores das Centrais de Regulação atribuem a garantia do acesso à disponibilidade de vaga pelo profissional executante do serviço. Para a Central Estadual de Regulação, os atores responsáveis pela garantia do acesso ao serviço de saúde necessário à usuária são as unidades de destino, uma vez que a central regula apenas a oferta da vaga, que é informada pela unidade assistencial por meio do profissional de referência. Para a Central de Regulação de Urgência, os atores responsáveis pela garantia do acesso são os médicos plantonistas das unidades.

Os entrevistados nas maternidades da rede e as unidades de pronto atendimento corroboram a afirmação de que a unidade executante do serviço é a responsável pela garantia da vaga. Algumas unidades atribuem essa prerrogativa ao profissional assistente, diretamente, uma vez que caberia a ele definir sobre a liberação da vaga, a exemplo do relato do gestor de unidade de pronto atendimento PA8, ao afirmar que
A central de regulação é uma intermediária, né, que só é uma organizadora do fluxo. [...] Quando a gente faz o contato, a gente faz de médico para médico. O médico daqui fala com médico plantonista de lá.

Outras unidades atribuem essa decisão ao núcleo interno de regulação, a exemplo da Maternidade M2: "o núcleo interno de regulação, eles são os responsáveis e eles têm que estar empoderados pra isso. Tanto o hospitalar quanto o ambulatorial".

No nível da atenção básica, o acesso aos serviços de saúde é organizado pelo gerente ou pelo enfermeiro da unidade, e o agendamento realizado na recepção. As entrevistas mostraram que, na atenção básica, o enfermeiro assistente assume um papel importante no acesso ao serviço de saúde, como observado na fala da gerente da UBS10: "A recepção marca. Ela [a enfermeira] monta a agenda, e a recepção faz conforme o agendamento que ela efetuou". Contudo, a atuação dessa categoria profissional revelou muita diversidade na execução das práticas nas diferentes unidades visitadas, inclusive na perspectiva da integração com os demais níveis da rede assistencial, ressaltando a capacidade de agência desempenhada por cada ator na rede assistencial. Exemplos podem ser observados na fala da coordenadora de enfermagem da UBS7, que relata uma organização aparentemente improvisada: "dificilmente fica sem vaga. A gente sempre dá um jeitinho de arrumar", diferindo do relato da enfermeira da UBS5, que descreve um acesso ao pré-natal mais sistematizado e acolhedor:

A primeira consulta se marca pra o serviço social. Tem o histórico social que ela colhe, apresenta a caderneta da gestante, faz tipo uma reunião de até 10 gestantes. Depois ela marca o retorno pra mim.

Na percepção das usuárias do serviço, entrevistadas na pesquisa, confirma-se que a atenção básica tem papel relevante no acesso à rede de assistência ao pré-natal e parto, principalmente em referência ao profissional enfermeiro como um apoio para viabilizar o 
pré-natal e orientar sobre o acesso aos serviços necessários. Registrando-se a fala da usuária G12.2, por quem ela procurava quando tinha alguma necessidade, "eu procurava a enfermeira de onde eu tava fazendo o pré-natal. Ela me dava os exames, eu marcava". Contudo, a maternidade, em especial, o serviço de pronto atendimento, também foi considerada como estratégica para o acesso mais rápido à unidade básica. Conforme afirma a gestante G9, "lá é mais difícil, porque a demora da entrega é grande, aí eu prefiro fazer aqui [na maternidade], que entrega mais rápido".

Nas entrevistas dos profissionais/gestores da rede, não foi possível captar o reconhecimento das usuárias como atores envolvidos no processo regulatório. No que pese, em várias situações, os profissionais colocam sob responsabilidade da usuária a busca do recurso solicitado, conforme observado nos relatos da coordenadora de enfermagem da UBS7

Existem os laboratórios que ofertam os exames pelo SUS, e aí as enfermeiras referenciam, orientam 'vá pra tal laboratório, é mais tranquilo, o resultado é mais rápido', [confirmados pela gerente da USF4] - ela que marca, não éde responsabilidade nossa. A gente encaminha, dá aquela ficha de referência e lá M4 acolhe, ou outra maternidade que foi encaminhada.

Quando questionados diretamente sobre quem é o responsável por viabilizar o acesso aos serviços de saúde, nenhum profissional ou gestor atribuiu essa função às usuárias. Possivelmente, o reconhecimento tácito de que a atribuição do acesso é de responsabilidade da rede assistencial, representada pelos profissionais e gestores, seja o motivo de não explicitar essa atribuição à usuária. A omissão dessa informação não significa que elas não estejam agindo ativamente, rotineiramente e sob a orientação dos profissionais que as assistem ou de outros contatos obtidos em suas próprias redes de relacionamentos, cujos exemplos foram observados, também, nas falas das gestantes, a exemplo de G5.4, que descreve sua experiência da seguinte forma:
Não foi encaminhamento. Ela me deu a requisição, eu fiquei sabendo que lá faz e aí eu fui pra lá. [...] A ultrassom eu fiz paga porque eu não sabia que a M2 fazia de graça. Aí a próxima eu vou fazer na M2.

As falas dos entrevistados destacam a capacidade de decisão atribuída ao profissional da assistência, com destaque para o médico no nível hospitalar, o que indica o poder desses agentes sociais na mobilização dos recursos na rede assistencial. Dessa forma, depreende-se que a ação regulatória não pode ser atribuição apenas da ação governamental. Ao contrário, é exercida em conjunto com diversos atores, individuais e organizacionais, presentes no território de abrangência da rede assistencial. Nesse cenário, atuam em interação profissionais da saúde - que solicitam os serviços de saúde às usuárias ou que realizam o serviço solicitado, representantes dos estabelecimentos que proveem os serviços de saúde -, que podem ser públicos ou privados, e as usuárias que demandam os serviços de saúde - ou seus representantes.

Estudos empíricos publicados na literatura nacional revelam que existe uma contradição entre a política de regulação - que preconiza uma rede normatizada, hierarquizada e racionalizadora -, e a operacionalização cotidiana da rede de atenção à saúde - que prevê um desenho horizontal, integrado e poliárquico dos serviços. Um elemento essencial para a prática regulatória, na perspectiva da rede, é a existência de mecanismos de negociação e pactuação entre os atores envolvidos no processo, uma vez que a interação entre estes é matéria prima da ação regulatória. Farias ${ }^{21}$, Fleury e Ouverney ${ }^{\mathbf{2 2}}$ defendem que para a regulação assistencial atuar como sistema logístico da RAS, as equipes gestoras devem ter como premissa a necessidade de criação de espaços permanentes de diálogo entre os profissionais das centrais de regulação e os profissionais que atuam nos diferentes níveis assistenciais da rede de saúde local. Esse diálogo poderá favorecer a construção conjunta de estratégias de integração sistêmica que apoiem a produção do acesso aos serviços da rede de forma viva e em ato. 


\section{Considerações finais}

A análise dos dados empíricos possibilitou compreender a conduta dos atores na ação regulatória como resultado de situações de oportunidades e de constrangimentos conferidas pela interação produzida com os demais atores envolvidos no contexto da rede assistencial de saúde.

Os resultados apontaram para a multiplicidade de atores envolvidos nessa prática social, individuais e organizacionais, profissionais que solicitam serviços de saúde e profissionais de saúde que produzem o serviço solicitado, gestores representantes dos entes estadual e municipal, representantes de unidades de saúde públicos e privados e as usuárias dos serviços, além dos reguladores, que, normativamente, seriam os responsáveis pela ação regulatória. Esse diverso coletivo de agentes, com intenções e motivações diferentes, produz cotidianamente a ação regulatória através de relações de interdependência, orientado pelas regras e pelos recursos disponíveis, que organizam a rede assistencial de pré-natal e parto no âmbito do SUS.

Essa característica ultrapassa a definição normativa de que a ação regulatória possui como agente principal o médico regulador, que possui atribuição legal de ordenar o acesso aos serviços de saúde na rede. Uma vez que existem diferentes atores envolvidos na oferta de serviços de saúde no SUS, a ação regulatória para viabilizar o acesso à saúde deve conseguir integrar em rede essa multiplicidade de atores para oferecer o recurso mais adequado às necessidades do usuário. Assim, é lícito concluir que a ação dos atores envolvidos nesse processo, em interação constante com outros atores, não é completamente controlável por meio de normas e regras formais. Essa configuração múltipla, dinâmica e complexa de agentes, com poderes para mobilizar recursos ou dificultar o acesso aos serviços de saúde, dificulta a comunicação integrada e pode produzir, de um lado, uma condição favorável às possiblidades particularistas de intermediação entre pontos de atenção, e, de outro, gerar constrangimentos à integração sistêmica da rede assistencial.

Conclui-se, ainda, que, adotando-se a concepção da regulação assistencial como uma prática social, ratifica-se a utilização de teorias sociais para sua compreensão e análise. Entre as possíveis ofertas teóricas, a teoria da estruturação pode contribuir enquanto ferramenta analítica para a explicação dos fenômenos sociais no âmbito do SUS, uma vez que possibilita o reconhecimento dos atores enquanto sujeitos reflexivos e dotados de capacidade de transformação, cuja prática está contextualizada pelo ambiente onde se desenvolve a ação.

\section{Colaboradoras}

Barbosa DSJ (0000-0001-9295-5880)* contribuiu para concepção, planejamento da pesquisa, análise e interpretação dos dados, e redação. Guimarães MCL (0000-0002-1311-8337)* contribuiu para concepção, planejamento da pesquisa e aprovação da versão final do manuscrito. 


\section{Referências}

1. Silva SF. Organização de redes regionalizadas e integradas de atenção à saúde: desafios do Sistema Único de Saúde (Brasil). Ciênc. Saúde Colet. 2011; 16(6):2753-2762.

2. Vilarins GCM, Shimizu HE, Gutierrez MMU. A regulação em saúde: aspectos conceituais e operacionais. Saúde debate. 2012; 36(95):640-647.

3. Oliveira RR, Elias PEM. Regulação em Saúde. In: Política e Gestão Pública em Saúde. São Paulo: Hucitec; 2011.

4. Mendonça CS, Reis AT, Moraes JC. A política de regulação do Brasil. Brasília, DF: Organização Pan-Americana da Saúde; 2006.

5. Gawryszewski ARB, Oliveira DC, Gomes AMT. Acesso ao SUS: representações e práticas de profissionais desenvolvidas nas Centrais de Regulação. Physis: 2012; 22(1):119-140.

6. Lobato LVC, Giovanella L. Sistemas de Saúde: origens, componentes e dinâmica. In: Políticas e Sistemas de Saúde no Brasil. Rio de Janeiro: Editora Fiocruz; 2008.

7. Cecilio LCO, Carapinheiro G, Andreazza R. Os mapas do cuidado: o agir leigo na saúde. São Paulo: $\mathrm{Hu}$ citec; 2014.

8. Giddens A. A constituição da sociedade. São Paulo: Martins Fontes; 1989.

9. Giddens A. Central Problems in Social Theory-Action, Structure and Contradiction in Social Analysis. Londres: Macmillan;1979.

10. Cecilio LCO. Escolhas para inovarmos na produção do cuidado, das práticas e do conhecimento: como não fazermos "mais do mesmo". Saúde Soc. 2012; 21(2):280-289.

11. Klijn EH, Koppenjan J, Termeer K. Managing ne- tworks in the public sector: a theoretical study of management strategies in policy networks. Public administration. 1995; 2(2):437-454.

12. Dimaggio PJ, Powell WW. Introduction. The new institutionalism in organizational analysis. Chicago: University of Chicago Press; 1991.

13. Brasil. Portaria $n^{0} 1.559$, de 1 de agosto de 2008. Política Nacional Regulação do Sistema Único de Saúde. Diário Oficial da União. 1 Ago 2008.

14. Santana JSS, Nascimento MAA. Pesquisa: métodos e técnicas de conhecimento da realidade social. Feira de Santana: Editora UEFS; 2010.

15. Silva JMB. A gestão do fluxo assistencial regulado no sistema único de saúde. Brasília, DF: Ministério da Saúde; 2011.

16. Oliveira LA, Cecilio LCO, Andreazza R, et al. Processos microrregulatórios em uma Unidade Básica de Saúde e a produção do cuidado. Saúde debate. 2016; 40(109):8-21.

17. Santos FP, Merhy EE. A regulação pública da saúde no Estado brasileiro: uma revisão. Interface-Comunicação, Saúde, Educação. 2006; 10(19):25-41.

18. Brasil. Ministério da Saúde, Departamento de Informática do Sistema Único de Saúde. homepage. Cadastro Nacional de Estabelecimentos de Saúde. [acesso em 2019 jan 3]. Disponível em: http://cnes. datasus.gov.br/.

19. Brasil. Ministério da Saúde. Portaria no 399 , de 22 de fevereiro de 2006. Divulga o Pacto pela Saúde 2006 - Consolidação do SUS e aprova as Diretrizes Operacionais do Referido Pacto. Diário Oficial da União. 23 Fev 2006.

20. Fleury S, Ouverney AM. Gestão de redes: a estratégia de regionalização da política de saúde. Rio de Janeiro: FGV Editora; 2007. 
21. Farias SF. Interesses estruturais na regulação da assistência médico-hospitalar do SUS. Recife. [tese]. Fundação Oswaldo Cruz, Centro de Pesquisas Aggeu Magalhães; 2009.

22. Fleury S, Ouverney AM. O sistema único de saúde bra- sileiro: desafios da gestão em rede. Revista de Gestão

dos Países de Língua Portuguesa. 2012; 11(2-3):74-83.

Recebido em 07/10/2020

Aprovado em 23/03/2021

Conflito de interesses: inexistente

Suporte financeiro: não houve 